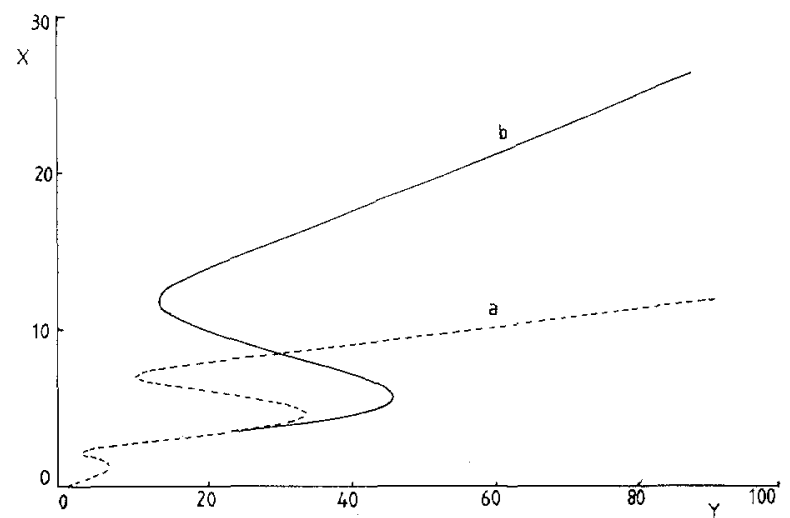

Fig. 5. Double bistability, $T_{1} / T_{2}=10, C=20, \Omega=24, \Phi=8$, (a) $\delta=$ $-0.1,(\mathrm{~b}) \delta=0.1$.

stability. The optical multistability may be useful for multilogic computers and signal processing.

\section{ACKNOWLEDGMENT}

The author is grateful to Dr. J. N. Elgin and Dr. J. A. Hermann for discussions.

\section{REFERENCES}

[1] E. Giacobimo, M. Devaud, F. Biraben, and G. Grynberg, "Dopplerfree two-photon dispersion and optical bistability in rubidium vapour," Phys. Rev. Lett., vol. 45, pp. 434-437, 1982.

[2] F. T. Arecchi and A. Politi, "Optical bistability in a resonant twophoton absorber," Lett. Al. Nuovo. Cimento, vol. 23, pp. 65-69, 1978.

[3] G. P. Agrawal and C. Flytzanis, "Two-photon double-beam optical bistability," Phys. Rev. Lett., vol. 44, pp. 1058-1061, 1980.

[4] J. A. Hetmann, "Stability condition for two-photon optical bistability in a ring cavity," Opt. Commun., vol. 37, pp. 431-436, 1981.

[5] D. F. Walls, C. V. Kunasz, P. D. Drummond, and P. Zoller, "Bifurcations and multistability in two-photon processes," Phys. Rev., vol. $24 \mathrm{~A}, \mathrm{pp} .627-630,1981$

[6] B. V. Thompson and J. A. Hermann, "Modified optical bistability in two-photon absorption," Phys. Lett., vol. 83A, pp. 376-378, 1981.

\title{
On the High Power Limit of the Laser Linewidth
}

\author{
AMNON YARIV AND KERRY VAHALA
}

\begin{abstract}
The quantum mechanical limit of the laser linewidth is shown to imply a residual constant linewidth rather than obey an inverse power dependence as is usually assumed.
\end{abstract}

$\mathrm{T}$ HE question of the limiting spectral linewidth of the field of a single mode laser was addressed in the early stages of the laser's development. Schawlow and Townes, the first to concern themselves with this problem, obtained [1]

$$
(\Delta \nu)_{\mathrm{laser}}=\frac{2 \pi h \nu\left(\Delta \nu_{1 / 2}\right)^{2}}{P} .
$$

Equation (1) was subsequently modified to [2]

$$
(\Delta \nu)_{\text {laser }}=\frac{2 \pi h \nu\left(\Delta \nu_{1 / 2}\right)^{2}}{P} \frac{N_{2}}{\left[N_{2}-N_{1}\left(g_{2} / g_{1}\right)\right]_{\text {th }}}
$$

where the factor

Manuscript received September 29, 1982. This work was supported by the Office of Naval Research and the National Science Foundation.

The authors are with the California Institute of Technology, Pasadena, CA 91125.

$$
\mu=\frac{N_{2}}{\left[N_{2}-N_{1}\left(g_{2} / g_{1}\right)\right]_{\mathrm{th}}}
$$

accounts for the fact that when $t_{1} \neq 0$, the finite population $N_{1}$ of the lower laser level requires a corresponding increase in $\mathrm{N}_{2}$ in order for the gain to remain equal to the loss. This increases the spontaneous emission noise power, which is proportional to $N_{2}$, and hence $(\Delta \nu)_{\text {laser }}$.

The common interpretation of (2) is that it predicts an inverse dependence of $(\Delta \nu)_{\text {laser }}$ on the power output $P$.

It is the purpose of this letter to point out that, according to (2), there should remain a residual laser linewidth, even as $P \rightarrow \infty$. This is due to the fact that, unless $t_{1}$ is zero, as $P$ increases, $N_{1}$ must increase since the increased net-induced transition rate into level 1 must equal in steady state $N_{1} / t_{1}$ the rate of emptying of level 1. This causes the population $N_{2}$ to increase in order to keep $N_{2}-N_{1}\left(g_{2} / g_{1}\right)$, and thus the gain, a constant. At sufficiently high values of $P, N_{2}$ becomes and stays proportional to $P$ and the ratio $N_{2} / P$ in (2) approaches a constant value, thus leading to a residual power independent linewidth. 
To obtain the power $(P)$ dependence of $\mu$, we solve the conventional laser rate equations

$$
\begin{aligned}
\frac{d N_{2}}{d t} & =R-\frac{N_{2}}{t_{2}}-\left[N_{2}-N_{1} \frac{g_{2}}{g_{1}}\right] W_{i} \\
\frac{d N_{1}}{d t} & =-\frac{N_{1}}{t_{1}}+\left[N_{2}-N_{1} \frac{g_{2}}{g_{1}}\right] W_{i}+\frac{N_{2}}{t_{2}} \\
\frac{d p}{d t} & =\left[N_{2}-N_{1} \frac{g_{2}}{g_{1}}\right] W_{i}-\frac{p}{t_{c}}
\end{aligned}
$$

where $N_{2}$ and $N_{1}$ are the level populations, $g_{1,2}$ are their degeneracies, $t_{1}$ and $t_{2}=t_{\text {spont }}$ are the lifetimes, $t_{c}$ is the passive resonator photon lifetime, $R$ is the pumping rate into level 2 , $W_{i}$ is the induced transition rate, and $p$ is the number of photons in the oscillating mode.

At equilibrium, $d / d t=0$, we can solve (4) to obtain

$$
\begin{aligned}
N_{2}-N_{1} \frac{g_{2}}{g_{1}} & =\frac{R\left[t_{2}-t_{1}\left(g_{2} / g_{1}\right)\right]}{1+W_{i} t_{2}} \\
N_{2} & =\frac{R t_{2}\left[1+W_{i} t_{1}\left(g_{2} / g_{1}\right)\right]}{\left(1+W_{i} t_{2}\right)}
\end{aligned}
$$

so that

$$
\mu=\frac{N_{2}}{\left[N_{2}-N_{1}\left(g_{2} / g_{1}\right)\right]_{\mathrm{th}}}=\frac{t_{2}}{t_{2}-t_{1}\left(g_{2} / g_{1}\right)}\left(1+W_{i} \frac{g_{2}}{g_{1}} t_{1}\right)
$$

where the subscript "th" indicates the value at threshold. The power output, including "wall losses" of the laser, is

$$
P=\left[N_{2}-N_{1} \frac{g_{2}}{g_{1}}\right]_{\mathrm{th}} W_{i} h \nu
$$

which, when used together with (6) in (2), gives

$$
\begin{aligned}
(\Delta v)_{\text {laser }}= & \frac{2 \pi h \nu\left(\Delta \nu_{1 / 2}\right)^{2}}{P}\left[\frac{t_{2}}{t_{2}-t_{1}\left(g_{2} / g_{1}\right)}\right] \\
& +\frac{c \Delta \nu_{1 / 2} \lambda_{0}^{2}}{8 \pi n^{3}(\Delta v)_{\text {gain }} V}\left[\frac{t_{1}}{t_{2}\left(g_{1} / g_{2}\right)-t_{1}}\right]
\end{aligned}
$$

where $\Delta \nu_{1 / 2} \equiv 1 / 2 \pi t_{c}$ and $\left(\Delta \nu_{\text {gain }}\right)$ is the linewidth of atomic transition responsible for the laser gain. $V$ is the mode volume. In obtaining (8), we use

$$
\left[N_{2}-N_{1} \frac{g_{2}}{g_{1}}\right]_{\text {th }}=\frac{8 \pi \nu^{2} n^{3}(\Delta \nu)_{\mathrm{gain}} V t_{2}}{c^{3} t_{c}} \quad\left(t_{2}=t_{\text {spont }}\right) .
$$

The first term on the right-hand side of (8) is the conventional Schawlow-Townes expression containing the inverse $P$ dependence. The second term is power independent and corresponds to a residual linewidth as $P \rightarrow \infty$.

To get an idea of the magnitudes involved, we consider the case of a $0.6328 \mu \mathrm{m} \mathrm{HeNe}$ laser with mirror reflectivities of $R=0.99$, a resonator length of $l=30 \mathrm{~cm}$, and take $t_{1} / t_{2}=0.1$. We obtain

$$
\Delta \nu_{1 / 2}(\mathrm{~Hz})=\frac{(1-R) c}{2 \pi n l}=1.6 \times 10^{6}
$$

and

$$
\Delta \nu_{\text {laser }}(\mathrm{Hz}) \simeq \frac{10^{-3}}{P(m \omega)}+3.8 \times 10^{-4} .
$$

The residual linewidth thus dominates at power levels exceeding a few milliwatts.

In a semiconductor laser the situation is more complicated. The dynamics of pumping are fundamentally different from those in a simple atomic laser. Charge neutrality will dictate that each injected electron is accompanied by the injection of a hole which would tend to clamp $N_{2}$ above threshold. For this reason we expect the above-described linewidth mechanism, if at all present, to have a negligible effect on the linewidth of a semiconductor laser.

\section{ACKNOWLEDGMENT}

The authors acknowledge useful discussions with S. Margalit.

\section{REFERENCES}

[1] A. L. Schawlow and C. H. Townes, "Infrared and optical masers," Phys. Rev., vol. 112, pp. 1940-1949, 1958.

[2] A. Yariv, Quantum Electronics, 1st ed. New York: Wiley, 1967. 